\title{
Gerontology research in Georgia
}

\author{
Teimuraz Lezhava $\cdot$ Jamlet Monaselidze $\cdot$ Tina Jokhadze $\cdot$ Nona Kakauridze • \\ Nodar Khodeli • Mamanti Rogava · Tamara Bochorishvili • Maia Gorgoshidze • \\ Dato Khachidze $\cdot$ Eteri Lomidze $\cdot$ Jaba Tkemaladze $\cdot$ Kote Chichinadze $\cdot$ \\ Marina Koridze $\cdot$ Rusudan Khukhuneishvili $\cdot$ Nato Zosidze $\cdot$ Marina Nagervadze \\ Tamara Buadze • Maia Gaiozishvili
}

Received: 23 April 2010/Accepted: 4 May 2010/Published online: 18 May 2010

(C) The Author(s) 2010. This article is published with open access at Springerlink.com

\begin{abstract}
Gerontology research carried out in different scientific centers of Georgia follows the basic directions of most work in this field: epidemiology, investigation of the mechanisms of aging, and finding ways to prevent senile pathologies and to prolong life. The genealogy and epidemiology of long-living peaple have been studied in areas with high occurrence of these people by considering the sex ratio and
\end{abstract}

T. Lezhava $(\bowtie) \cdot$ T. Jokhadze · N. Khodeli .

T. Buadze $\cdot$ M. Gaiozishvili

Department of Genetics, Tbilisi State University, Chavchavadze ave.1, 0128 Tbilisi, Georgia

e-mail: lezhavat@yahoo.com

J. Monaselidze · M. Gorgoshidze - D. Khachidze ·

E. Lomidze

Institute of Physics, Tamarashvili, 6, 0177 Tbilisi,

Georgia

N. Kakauridze · M. Rogava · T. Bochorishvili Georgian National Centre of Therapy, Chachava 3, Tbilisi, Georgia

M. Koridze · R. Khukhuneishvili · N. Zosidze ·

M. Nagervadze

Batumi State University, Ninoshvili 35, Tbilisi, Georgia

J. Tkemaladze

Gergian Sistemic Research Center, pr. Vaja Pshavela

18A, 0160 Tbilisi, Georgia

K. Chichinadze

Institute of Physiology, Gotua 14, 0160 Tbilisi, Georgia social status of the long-living, the influence of environmental factors, and the development of senile pathologies. According to the centrosome (centriole) model of aging, the centrosomes and the cytoskeleton, important structures in cellular differentiation and morphogenesis, may be involved in the initiation of the replication senescence mechanism. Our analysis of genetic studies shows that progressive chromosome heterochromatinization (condensation of eu- and heterochromatin regions) occurs in aging. Decreases in the repair processes and increases in the frequency of chromosome aberrations during aging are secondary to this progressive chromosome heterochromatinization. Chromosome heterochromatinization is a key factor in aging but may be reversible under the influence of bioregulators, some chemical substances, and heavy metal salts. The study of chromosome heterochromatinization may provide clues to the potential for prolonging the human lifespan.

Keywords Aberration - Aging - Centrosome . Chromosome $\cdot \mathrm{Co}^{2+}$. Epidemiology .

Heterochromatin · Heterochromatinization . Microcalorimetry
Abbreviations
Ag-positive
Silver stained
C-band
Band of pericentromeric heterochromatin
CVD
Cardio-vascular disease
DSM 
NOR Nucleolus organizer regions

SCE Sister chromatin exchange

\section{Introduction}

Georgia is a country of long-living people, and the proportion of the population comprising persons aged 90 years and older is quite high. Areas within Georgia with a very high frequency of centenarian individuals include the mountainous Svaneti region and the costal Black Sea region of Adjara. These two areas differ markedly from each other in geographic and climatic conditions, and in lifestyle. Because of the high proportion of older adults, gerontology research is a priority in Georgia. Within the framework of the Academy of Sciences, the Ministry of Health, and some higher education institutions, gerontology centers and independent groups of scientists have been researching for many years the epidemiology of aging, the underlying mechanisms, and ways to prevent senile pathologies and to prolong life. Their activities are coordinated by the Gerontological Society of Georgia, which is chaired by professor N. Kipshidze.

\section{Epidemiological investigations}

Based in Batumi State University, a team has been studying the epidemiology of aging in the Black Sea coastal areas of Khulo, Keda, Shuakhevi, and Khelvachauri. They are focusing on the sex ratio, social status, and lifestyles of the long-living and influences of the environment, and data on blood group antigens have been collected. Observations of the life conditions and characteristics of these people show that they are distinguished for their physically active and healthy lifestyles and diet, wide interests and curiosity, and normal daily regime. These longliving people are frequently exposed to fresh air, enjoy life, and are delightful and brisk.

More women than men make up the long-living. Most of these women married at age 18-30 years, and $76.1 \%$ gave birth at age $30-54$ years. A high proportion of them lived most of their life with a spouse and most of these long-living people have long-living offspring, some aged almost 80 years. More than $90 \%$ of the long-living respondents "felt their age" only after they turned 80, 76.4-78.5\% had long-living parents, and $76.9 \%$ consider themselves healthy. The diets varied, and equal proportions ate a vegetarian diet and mixed diet; most preferred lowcalorie foods.

Analysis of blood group antigens showed a high frequency for groups $\mathrm{O}(1)(56 \%)$ and A(II) (36\%). The frequency of $\mathrm{C}, \mathrm{D}$, and $\mathrm{E}$ antigens was also higher among the long-living compared with the general population. Analysis using the Kell blood group system showed that $6 \%$ were $\mathrm{K}+$ and $94 \%$ were $\mathrm{K}-; \mathrm{K}+$ was more frequent in the long-living than in the general population. Analysis according to the $\mathrm{MN}$ blood group system showed that $82 \%$ were $\mathrm{M}+, 4 \%$ were $\mathrm{N}+$, and $14 \%$ were $\mathrm{M}+\mathrm{N}+, \mathrm{M}+$ was more frequent in the long-living than in the general population (Koridze et al. 2009).

\section{Clinical and genealogical investigations}

A group of scientist-physicians at the Georgian National Centre of Therapy have been studying various aspects of longevity and aging using genealogical and biochemical markers such as blood total cholesterol and triglyceride concentrations. They have shown that inheritance plays a role in longevity. Genealogical and biochemical data have been obtained from senile individuals to investigate the pathologies related to aging such as atherosclerosis. To clarify the role of genetic influence in aging and longevity, the clinical and genealogical characteristics of 54 patients of both sexes have been studied; 34 were long-living ( $>90$ years) and 20 were elderly ( $70-90$ years).

Clinical and laboratory investigations were carried out in accordance with the World Health Organization's recommendations. The total content of cholesterol and triglycerides was assessed by the enzymemeasuring method. Sing a genealogical questionnaire, data have been collected on morbidity, mortality, health condition, and cardiovascular disease (CVD) in 206 patients and their parents and siblings. The fathers of the long-living had lived longer than the fathers of the elderly patients, thus confirming the importance of parental-line inheritance to longevity. We have also found a genealogical relationship between the health of the long-living (e.g., absence of CVD) and the genealogical anamnesis about their parents. Stability of lipid metabolism was observed in 
the long-living and the elderly; total cholesterol concentration was lower in the long-living than in the elderly. In both groups, total cholesterol content correlated with the rate of the CVD. These data confirm the importance of genetic factors to longevity (Kakauridze et al. 2008).

The capacity of peripheral blood $\mathrm{T}$ lymphocytes to respond to irradiation was studied in patients with hypertrophic cardiomyopathy (Jokhadze T., Rogava M.) To induce the adaptive response, Cs B7 gamma rays were used. The whole blood was irradiated by a stimulating dose $(0.2 \mathrm{~Gy})$ and by a main-injurious dose ( 2 Gy) for $2.5 \mathrm{~h}$. The fractional irradiation effect was estimated by the frequency of the structural and numeric chromosomal abnormalities in cultured lymphocytes. The T lymphocytes of the examined patients maintained the capacity to respond to the radiation. Preliminary irradiation with the stimulating dose decreased the frequency of lymphocytes with chromosomal aberrations, and such aberrations were induced only by the main dose from $19.0 \%$ to $9.5 \%$. Irradiation by the separate stimulating and injurious dose fractionation caused different responses of the chromosome parameters such as aneuploidy frequency and chromosomal aberrations in patients compared with the control group. The authors explained the observed effects in terms of the specific modification of chromatin in lymphocytes of patients with cardiomyopathy (Jokhadze et al. 2005; Kakhidze et al. 2006; Jokhadze et al. 2007).

\section{Role of the centrosome in aging}

Scientists at the Georgian Centre of System Analysis and the Institute of Physiology have been researching the mechanisms underlying the aging process, in particular the centrosome hypothesis. This hypothesis focuses on the structures that control differentiation, morphogenesis, and replication in somatic cells of many-celled animals - the centrosome and cytoskeleton. Programming of the mechanisms of such event is influenced by the cytoskeleton and perhaps by si RNA related to the centrosome. If the given functions are related to the centrioles, then the cells of multicellular bodies should show an initial absence of centrioles (e.g., higher plant cells and zygotes and the first blastomeres of some animals), cytoskeleton (e.g., embryonic stem cells), or the de novo appearance of centrioles (e.g., zygotes and the early blastomeres of some animals). Such cells will appear to be totipotent and will lack replicate senescence, indicating that the permanent or temporary absence of the structure that determines division lacks the differentiation processes. Although certain damage to the centrioles in the cytoskeleton (e.g. in tumor sells) makes them totipotent, to morphogenetic status of such cells in comparison with the totipotential cells is not equal to zero. However, such transformation can suppress the initiation of the aging mechanism induced by these structures and thus make such cells replicatively "immortal" (Tkemaladze and Chichinadze 2005; Chichinadze and Tkemaladze 2008).

\section{Genetic aspects of aging}

A group of scientists from Tbilisi State University in close cooperation with the scientists of the Institute of Physics have been studying the genetic aspects and pathologies of aging. Their work also focuses on finding ways to prevent age-related pathologies and to apply the general mechanisms of aging toward prolonging life.

The aging process is programmed in the genome of each organism and is manifested late in life. Any change in normal homeostasis, particularly any further loss of the cell function with aging, occurs in the functional units of the chromatin domains.

Modification of the chromatin structure and function by hetero- or deheterochromatinization occurs throughout life and plays a pivotal role in the irreversible process in aging by affecting gene expression, replication, recombination, mutation, repair, and programming (Gilson and Magdinier 2009).

Heterochromatin is divided into two main forms according to their distinct structural functional dynamics: constitutive heterochromatin $(\mathrm{CH})$ and facultative heterochromatin $(\mathrm{FH})$. $\mathrm{CH}$ refers to the regions that are always maintained as heterochromatin; these span large portions of the chromosome and have a structural role. $\mathrm{CH}$ regions contain few genes and are located primarily in pericentromeric regions and telomeres. $\mathrm{FH}$ refers to those regions that can be formed as heterochromatin in a certain situation but can revert to euchromatin once required. FH can span from a few kilobases to a whole chromosome and generally includes regions with a high density of genes (Prokofieva-Belgovskaya 1986; Vaquero 2009). 
We have used differential scanning microcalorimetry to produce a calorimetric curve in cultured human lymphocytes over the temperature range 38$130^{\circ} \mathrm{C}$ (Monaselidze et al. 2006, 2008). The heating process produced clear and reproducible endothermic heat absorption peaks. We found that an endothermic peak at $T_{\mathrm{m}}=104 \pm 1{ }^{\circ} \mathrm{C}$ corresponds to melting of $30 \mathrm{~nm}$-thick fibers, which represents the most condensed state of chromatin in interphase nuclei (heterochromatin), and that an endothermic peak at $96 \pm 1{ }^{\circ} \mathrm{C}$ corresponds to melting of $10 \mathrm{~nm}$-thick filaments (euchromatin or chromatin). The chromatin heat absorption peaks VI and VII changed significantly with age. In particular, in the shifted endotherms VI and VII, the temperatures increased by $2^{\circ} \mathrm{C}$ and $3^{\circ} \mathrm{C}$ accordingly in old age (80-86 years). Additional condensation of the eu- and heterochromatin was demonstrated by an increase in $T_{\mathrm{m}}$ by 2 and $3^{\circ} \mathrm{C}$ in comparison with the meddle age (25-40 years). These prominent changes in chromatin stability indicated transformation of eu- and heterochromatin in condensed chromatin (heterochromatinization).

One of the potential epigenetic mechanisms is heterochromatinization of chromatin within the region of the genome containing a gene sequence, which inhibits any further molecular interactions with that underlying gene sequence and effectively inactivates that gene (Ellen et al. 2009). The chromatin peak behavior described above shows progressive heterochromatinization of lymphocyte chromosomes from old individuals and confirms previously reported data (Lezhava 1984, 2001, 2006). These significant changes in chromatin stability in old age indicate that the aging process involves transformation of the euand heterochromatin into condensed forms and that further compaction or progressive heterochromatinization occurs during aging.

\section{Correlation between mutation, repair and heterochromatinization of chromosomes in aging}

Progressive heterochromatinization of chromosome regions observed during aging correlates with the greater frequency of chromosome aberrations and the reduced intensity of reparative events. Chromosome alterations have been studied in 70 individuals aged 80-114 years (30 women and 40 men). In these samples, the percentages of aberrant metaphase and chromosomal aberrations were $4.08 \pm 0.41 \%$ in women and $5.15 \pm 0.45 \%$ in men; these values are significantly higher than the control levels (aged 2540 years) of $1.8 \pm 0.42 \%$ and $2.15 \pm 0.35 \%$, respectively (Lezhava 2006).

Our studies have also demonstrated a marked decline in the unscheduled DNA synthesis (repair) rates in 80-90 year old individuals in response to UV irradiation at a dose of $10-15 \mathrm{~J} / \mathrm{mm}(P<0.03$, $P<0.01$ respectively) compared with the middleaged individuals. These data suggest that human lymphocytes from older people (aged 80 years or more) have a significantly reduced capacity for unscheduled DNA synthesis-excision repair (Lezhava 2006).

To explain the prevalence of the accumulation of damage in heterochromatin in the heterochromatinization regions, it has been assumed that the repair of lesions capable of causing aberrations is possible only in those areas of DNA that are actively involved in transcription and that are within physically accessible reach of reparative enzymes, i.e. in euchromatin areas (Prokofieva-Belgovskaya 1986). Assuming that heterochromatinized regions are inaccessible to reparative enzymes and therefore the number of cells with chromosome aberrations profoundly affects the functioning of the genome in old age. Our results indicate that decreases in the repair processes and increases in the frequency of chromosomal aberrations in aging are secondary to the progressive heterochromatinization and that chromosome heterochromatinization is a key factor in aging.

\section{Heterochromatin and pathology}

Heterochromatinization progresses with aging and can deactivate many previously functioning active genes. It blocks certain stages of normal metabolic processes of the cell, which inhibits many specific enzymes and leads to aging pathologies. The action of genetic systems reveals general rules in the behavior of such systems, such as the connection between the structural and functional interrelationships between the "directing" and "directed" structures. In the respect, it should be noted that heterochromatinized regions in chromosomes can reverse. Many physical and chemical agents, hormones and peptide bioregulators cause deheterochromatinization (decondensation) (Khavinson et al. 
2002, 2003; Lezhava and Bablishvili 2003; Lezhava et al. 2004). Such actions may be helpful in the treatment of diseases of aging.

We have demonstrated also that $\mathrm{Co}^{2+}$ ions alone and in combination with the bioregulator Livagen can reverse the deheterochromatinization of precentromeric and telomeric heterochromatin to normalize the telomere length in cells from old individuals (Lezhava and Jokhadze 2007; Lezhava et al. 2008).

The proposed genetic mechanism responsible for heterochromatin remodeling (hetero- and deheterochromatinization) of senile pathogenesis highlights the importance of external and internal factors in the development of diseases and may lead to the development of therapeutic treatment.

We believe that the next step in research should be:

1. genetic and epidemiological investigations of the mechanisms of aging in individuals aged 100 years and older;

2. such knowledge will help develop ways to prevent senile pathologies;

3. and to prolong life.

Open Access This article is distributed under the terms of the Creative Commons Attribution Noncommercial License which permits any noncommercial use, distribution, and reproduction in any medium, provided the original author(s) and source are credited.

\section{References}

Chichinadze K, Tkemaladze J (2008) Centrosomal hypothesis of cellular ageing and differentiation. Adv Geront 21:367371

Ellen T, Kluz T, Harder M, Xiong J, Costa M (2009) Heterochromatinization as a potential mechanism on Nickelinduced carcinogenesis. Biochemistry 48:4626-4632

Gilson E, Magdinier F (2009) Chromosomal position effect and aging. In: Epigenetics and aging (vol 2). Springer, New York, pp 151-175

Jokhadze T, Lezhava T, Kakhidze Kh, Rogava M, Tabatadze N (2005) Chromosome instability in patients with different form cardiomiopathy and their relatives. Georg Med News 12:134-138

Jokhadze T, Buadze T, Dvalishvili N, Lezhava T (2007) Variability of radiation-induced adaptive response in old age individuals and their correction by peptide bioregulatorlivagen. Georg Med News 7-8:50-54

Kakauridze N, Guchua E, Mgaloblishvili M (2008) Genetics aspects of longevity and aging. Georg Med News 10: $71-76$
Kakhidze Kh, Jokhadze T, Rogava M, Bochorishvili T, Buadze T (2006) Allergol Immunol 7:536-538

Khavinson V, Lezhava T, Monaselidze J, Jokhadze T, Dvalishvili N, Bablishvili N, Riadnova I (2002) Effect of Livagen on chromatin in lymphocytes from old people. Bull Exp Biol Med 134:389-392

Khavinson V, Lezhava T, Monaselidze J, Jokhadze T, Dvalishvili N, Bablishvili N (2003) Peptide Epitalon activates chromatin at the old age. Neuroendocrinol Lett 24:329333

Koridze M, Khukhunaishvili R, Zosidze N (2009) Biogerontological study of population in some districts of Ajara Autonomous Republic. Georg Medl News 6:53-56

Lezhava T (1984) Heterochromatinization as a key factor of aging. Mech Ageing Dev 28:279-288

Lezhava T (2001) Chromosome and aging: genetic conception of aging. Biogerontology 2:253-260

Lezhava T (2006) Human chromosomes and aging. From 80 to 114 years. New York "Nova biomedical" 177

Lezhava T, Bablishvili N (2003) Reactivation of heterochromatin induced by sodium hydrophospate at the old age. Proc Georg Acad Sci Biol Ser B 1:1-5

Lezhava T, Jokhadze T (2007) Activation of pericentromeric and telomeric heterochromatin in cultured. Ann N Y Acad Sci 1100:387-399

Lezhava T, Khavinson V, Monaselidze J, Jokhadze T, Dvalishvili N, Bablishvili N, Barbakadze Sh (2004) Bioregulator Vion-induced reactivation of chromatin in cultured lymphocytes from old people. Biogerontology 4:73-79

Lezhava T, Monaselidze J, Jokhadze T (2008) Decondensation of chromosmes heterochromatinization regions by effect of heavy metals and bioregulators in cultured lymphocytes from old individuals. In: Proceedings of the 10th International Symposium of Metal Ions in Biology and Medicine, Bastia France May 19-22. Edited by Philippe Collery 10:569-576

Monaselidze J Bregadze V, Barbakadze Sh, Majagaladze G, Khachidze D, Kiladze M, Kuchadze Z, Lezhava T, Jokhadze $T$ (2008) Influence of metal ions of thermodina stability of leukemic DNA in vivo. Microcalorimetri investigation. In: Proceeding of the 10th international symposium of metal ions in biology and medicine, Bastia France May 19-22. Edited by Philippe Collery 10:451-457

Monaselidze J, Abuladze M, Asatiani N, Kiziria E, Barbakadze Sh, Majagaladze G, Iobadze L, Tabatadze I, Holman HoiYing, Sapojnikova N (2006) Characterization of chromium-induced apoptosis in cultured mammalian cells. A different scanning calolorimetry study. Thermochem Acta 441:8-15

Prokofieva-Belgovskaya A (1986) Heterochromatin regions of chromosomes. M "Nauka" 431

Tkemaladze J, Chichinadze K (2005) Centriolar mechanisms of differentiation and replicative aging of higher animal cells. Biochemistry (Moscow) 70:1288-1303

Vaquero A (2009) The conserved role of sirtuims in chromatin regulation. Int J Dev Biol 53:303-322 